\title{
Determinación de Presiones Intraorales en Compartimientos Biofuncionales de Pacientes Desdentados Totales
}

\author{
Determination of Intraoral Pressures on Biofunctional Compartments in Edentulous Patients
}

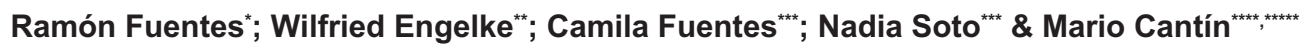

FUENTES, R.; ENGELKE, W.; FUENTES, C.; SOTO, N. \& CANTÍN, M. Determinación de presiones intraorales en compartimientos biofuncionales de pacientes desdentados totales. Int. J. Odontostomat., 8(2):151-157, 2014.

RESUMEN: El objetivo fue describir las diferentes presiones intraorales que se presentan en los espacios interoclusal vestibular (EIO) y subpalatino (ESP) bajo distintas condiciones biofuncionales en pacientes adultos desdentados totales maxilares. Fueron seleccionados 15 pacientes en la Clínica Odontológica Docente Asistencial, Universidad de la Frontera (Temuco, Chile). La medición de presiones intraorales se realizó con un dispositivo digital GMH 3156, mediante los métodos de cánulas (control) y escudo oral. Se realizó una medición de calibración por 15 minutos y luego cuatro mediciones funcionales: B1 (condición abierta; 0-25 s); B2 (condición semiabierta; 35-85 s); B3 (condición cerrada; 95-145 s) y B4 (presión bajo deglución; 150-165 s). Con el escudo oral, la Fase B1 mostró en el ElO una presión constante entre 0-4 mBar y el ESP entre -1 y -3 mBar. En la Fase B2, el EIO llegó a 10 mBar y el ESP entre 0 y -1 mBar. La Fase B3 mostró en el EIO oscilaciones entre 0 y 20 mBar, y en el ESP un aumento hasta 20 mBar. En la Fase B4, el EIO presentó un peak de 40 mBar, mientras que el ESP un peak desde 20 hasta -140 mBar. La formación de dos compartimientos cerrados con presión intraoral negativa también ocurre en pacientes edéntulos, donde el ESP muestra cambios de presión negativa mayores al EIO. Patrones regulares de presión intraoral en espacios biofuncionales se observan en más del $50 \%$ de los casos.

PALABRAS CLAVE: pacientes desdentados, presión intraoral, presión negativa, modelo biofuncional, compartimentos intraorales funcionales.

\section{INTRODUCTION}

Existe una estrecha relación de balance entre tejidos duros orales (dientes y hueso alveolar) y tejidos blandos (mejillas, labios y lengua) (Abrams, 1963; Proffit, 1978; Knösel et al., 2010), la cual conforma una matriz capsular cerrada, compuesta por compartimientos biofuncionales intraorales, donde se genera una diferencia de presión con respecto al medio atmosférico externo, conocida como presión intraoral (Moss \& Salentijn, 1969; Knösel et al.). Con la perdida dentaria (por caries, enfermedad periodontal, trauma, etc.) y posterior reabsorción del hueso alveolar, el equilibrio se pierde y provoca la alteración de los tejidos blandos, así como la imposibilidad de generar presiones intraorales negativas de manera adecuada. Esto se puede correlacionar con una masticación y mímica muscular hipotónica o una baja fuerza del labio, manifestación clínica y efecto de un equilibrio perturbado de las fuerzas, que provoca cambios en las presiones intraorales de los compartimientos biofuncionales (Proffit; Knösel et al.; Jung et al., 2011), así como desestabilización del paladar blando y ronquido primario o alteraciones en los mecanismos de sellado y estabilidad de las prótesis maxilares completas.

Engelke (2003) mediante la maniobra de reposicionamiento de la lengua, reportó la formación voluntaria de presión negativa intraoral (cerrada en posición de reposo), logrado por la influencia de un

\footnotetext{
Centro de Investigación CIMOFIR, Facultad de Odontología, Universidad de La Frontera, Temuco, Chile.

Dentistry Center, Department of Maxillofacial Surgery, Georg-August-Universität, Göttingen, Alemania.

*** Alumna Escuela de Odontología, Facultad de Odontología, Universidad de La Frontera, Temuco, Chile.

Doctorado en Ciencias Morfológicas, CEMyQ, Centro de Investigación CIMA, Facultad de Odontología, Universidad de La Frontera, Temuco, Chile.

****** Centro de Investigación en Ciencias Biomédicas, Universidad Autónoma de Chile, Temuco, Chile.

Financiado por Proyecto DIUFRO DI12-0051, Dirección de Investigación, Universidad de La Frontera, Temuco, Chile.
} 
modo de respiración exclusivamente nasal. Además, mediante el uso de dispositivos orales o escudos orales, se ejercen influencia sobre el equilibrio de las fuerzas del medio ambiente intraoral y por tanto, espacios biofuncionales por un estado cerrado y presión subatmosférica, que sirven como factor de estabilización biomecánico, no solo a expensas de la actividad neuromuscular de las estructuras orofaciales, sino que también a las posturas orales biofuncionales, las que finalmente determinan el equilibrio biomecánico de las fuerzas por elementos externos e internos (Engelke et al., 2006, 2007, 2010, 2011).

Hasta ahora, no está claro en qué medida los efectos del sistema operativo pueden ser atribuidos a las fuerzas activas neuromusculares del tejido blando por sí solo o a la presión física intraoral. Si esto último es cierto, el uso de estos dispositivos pueden influenciar la posición lingual o trastornos funcionales, mediante la generación conciente o inconciente de presiones negativas intraorales, similares a los ejercicios aplicado con éxito en el tratamiento para los ronquidos mediante el uso de dispositivos intraorales como los escudo oral descrito por Engelke et al. (2006). Por tanto, la detección de cambios provocados por los dispositivos en los niveles de la presión intraoral negativa proporcionaría la base para el desarrollo de enfoques de tratamiento para lograr una normalización permanente de posibles desbalances en el equilibrio de fuerzas intraorales, principalmente en pacientes desdentados (Proffit; Engelke et al., 2006, 2010; Knösel et al.).

El objetivo de este estudio fue evaluar la dinámica de la presión atmosférica y describir de las diferentes presiones intraorales que se presentan en los espacio interoclusal vestibular y subpalatino en diferentes condiciones biofuncionales medidas mediante el uso de dispositivos orales en pacientes adultos y adultos mayores desdentados totales maxilares.

\section{MATERIAL Y MÉTODO}

Se realizó un estudio de serie de casos, de tipo descriptivo y corte transversal. El universo de estudio se seleccionó desde la Clínica Odontológica Docente Asistencial, Universidad de la Frontera (Temuco, Chile). El tamaño muestral se ajustó a estudios previos. Fueron seleccionados sujetos adultos y adultos mayores de Temuco de ambos sexos, que cumplieran los siguientes criterios de inclusión: dentados totales maxilares y portadores de prótesis dental acrílica removible total. Se excluyeron casos de pacientes con presencia de lesiones o patologías en la mucosas, obstrucción de las vías respiratorias (evaluación anamnésica), respiración nasal clínicamente perturbada en reposo, presencia de enfermedades o trastornos motores, que consuman neurolépticos o enfermedades de flujo salival. El estudio fue aprobado por el comité de ética de la Facultad de Medicina de la Universidad de La Frontera. En todos los casos, la participación fue totalmente voluntaria y se firmó un consentimiento informado.

En cada sujeto se realizó la medición de las presiones intraorales utilizando como control el método con cánulas descrito por Engelke et al., (2010) y luego con un dispositivo oral único, de tipo escudo oral. En ambos métodos, la presión intraoral se determinó con el mismo sensor de presión.

Medidor de presión. Se utilizó un medidor de presión digital (PDA) portátil (GMH 3156, Greisinger electronic, Germany) conectado a dos sensores de presión relativa piezo resistente (GMSD 350 MR; GmbH Greisinger electronic, Regenstauf, Germany) con un rango de medición de 500 mbar (rel.) (100 a -400 mbar) y una resolución de 0,1 mbar de presión relativa. Cada sensor se conectó independientemente a un tubo flexible de PVC (4 mm de diámetro interno) (Engelke et al., 2010; Knösel et al.).

Método de Control. Se utilizaron dos accesorios terminales flexibles diferentes, un catéter intravenoso de polietileno semi-flexible (20Gx1.00", BD Cateter InsyteTM, Becton Dickinson Ind. Cir. Ltda., Brasil) y un eyector de saliva transparente de PVC, con tapa dental de aspiración aire-permeable, de $15 \mathrm{~cm}$ de longitud (Fig. 1A), conectados a los sensores de presión. La medición de la presión atmosférica se realizó intraoralmente en dos zonas a través del extremo libre del catéter intravenoso y el eyector: (1) en el espacio interoclusal del compartimiento vestibular (EIO) y (2) en el espacio sub-palatino (ESP) entre la bóveda palatina y la superficie de la lengua, respectivamente. Para medir el EIO, se colocó el eyector por la mucosa bucal de la mejilla con la tapa de aspiración permeable en la zona vestibular contigua a la región premolar. Se utilizaron los molares inferiores como parámetro de posicionamiento o en caso de edentulismo total mandibular, fue la región posterior a los frenillos vestibulares laterales. Para medir el ESP, el catéter se posicionó intraoralmente hasta llegar con la a la zona central (plano mediano) de la bóveda palatina, a $3 \mathrm{~cm}$ 


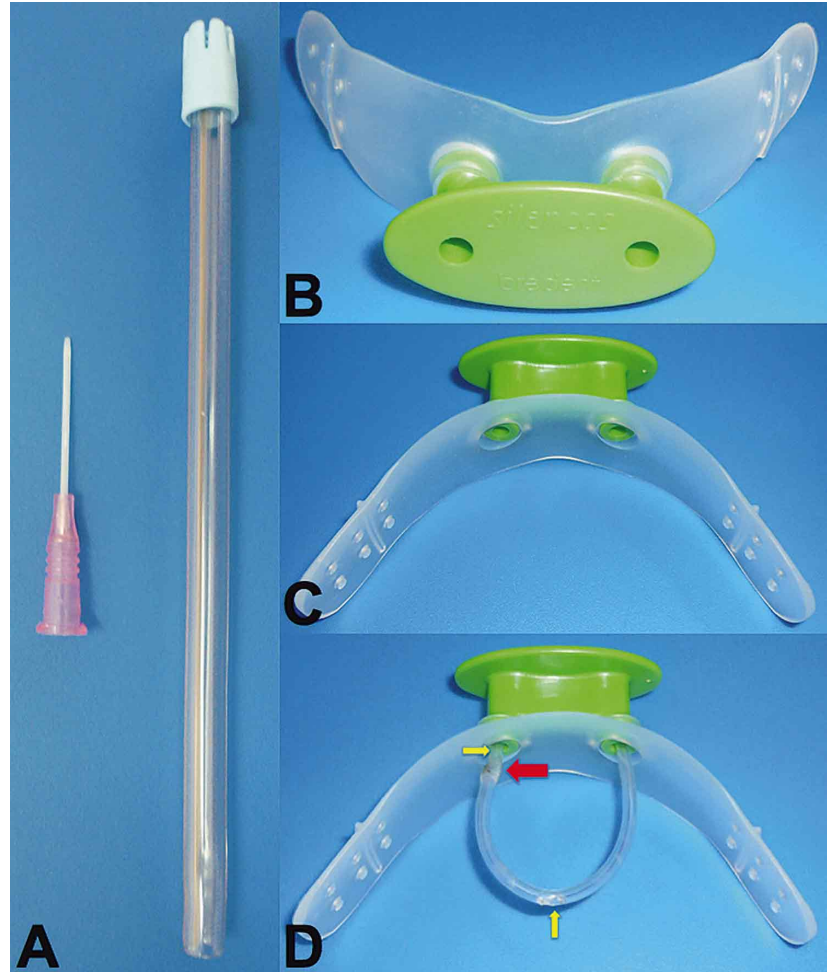

Fig. 1. A. Catéter intravenoso de polietileno semi-flexible (izquierda) y eyector de saliva transparente de PVC (derecha) que son conectados al tubo flexible de PVC de los sensores por un extremo, y el otro extremo se dirige intraoralmente para realizar la medición de la presión atmosférica. Escudo oral Activador de vació silencos. Se observa una visión anterior del dispositivo con las perforaciones externas (B) y visión superior del dispositivo (C). D. Escudo oral con arco de tubo flexible. La flecha roja indica la zona donde se sello el tubo; las flechas amarillas indican los puntos donde se realizaron las perforaciones del tubo con el fin de comunicar con la cavidad oral y poder registrar las presiones.

desde el reborde alveolar al tercer pliegue palatino transverso, con el fin de reducir la interferencia con el dorso de la lengua. Para estabilizar el catéter, se adaptó mediante silicona al reborde alveolar.

Método de escudo oral. Se utilizó un dispositivo oral de tipo escudo oral (Activador de vacío silencos, Bredent, GmbH \& Co.KG, Germany) (Fig. 1B-C). El escudo oral fue conectado en cada una de las perforaciones externas al tubo flexible de PVC proveniente del sensor de presión. Se confeccionó un arco flexible de PVC (2 mm diámetro interno, $10 \mathrm{~cm}$ de longitud), donde cada uno de los extremos se conectó al tubo del sensor. El arco se selló a $5 \mathrm{~mm}$ de la perforación interna izquierda del escudo intraoral. Finalmente, se realizaron dos perforaciones en el arco, la primera entre la perforación interna y la zona sellada, la segunda en el punto más convexo del arco, por su cara externa (Fig. 1D). Estas perforaciones coinciden y permiten la medición de presión en el EIO y ESP.

Medición de la presión intraoral. Todas las mediciones fueron llevadas a cabo por un solo operador clínico entrenado. Cada paciente se ubicó en el sillón dental sentado en posición vertical, con una posición natural de la cabeza (Archer \& Vig, 1985) y se retiró su prótesis maxilar. Tanto para las mediciones de control y con escudo oral, los sensores se ubicaron frente a él, a altura de la cavidad oral. Luego explicar el procedimiento y adaptar en boca cada dispositivo, se les solicitó que siguieran las instrucciones indicadas por el operador. Se realizó medición de presión primero con el método control y luego el método de escudo oral, ambos en dos fases.

Fase A: Calibración. Se realizó la medición como control de presión por un periodo de total de 15 minutos, a fin de evaluar los efectos de los dispositivos intraorales para medir el EIO y ESP. Los pacientes fueron instruidos a mantener los dispositivos presionados suavemente con los labios, tragar y respirar de manera normal durante el período, a fin de que el paciente mantenga su biofuncionalidad natural.

Fase B: Medición funcional. Fase B1. Medición de la presión bajo condición condición abierta (CA), desde 0 a 30 segundos. A los pacientes se les solicitó que abrieran un poco los labios y respiraran tranquilamente.

Fase B2. Medición de la presión bajo condición condición semi-abierta (CSA), desde los 30 a 90 segundos. A los pacientes se les solicitó que cerraran los labios suavemente y siguieran respirando tranquilamente. La duración se registro durante 60 segundos.

Fase B3. Medición de la presión bajo condición compartimento cerrado (CC), desde los 90 a 150 segundos. Al paciente se le solicitó colectar saliva succionado la cara interna de las mejillas; luego que tragara forzadamente la saliva y luego respirara tranquilamente. La duración de la condición cerrada fue de 60 segundos.

Fase B4: Medición de la presión bajo Deglución (PD), desde los 150 a 165 segundos. A los pacientes se les solicitó tragar su saliva de manera normal.

Análisis de datos. Para la evaluación de los datos, se seleccionarán intervalos de tiempo, a fin de eliminar las fases transitorias entre las diferentes condicio- 
nes, quedando determinados los siguientes intervalos: Fase B1 (CA): 0-25 s; Fase B2 (CSA): 35-85 s; Fase B3 (CC): 95-145 s y Fase B4 (PD): 150-165 s.

Los datos de las fases $A$ y $B$ de las mediciones control y con escudo oral fueron procesadas mediante el Software operativo GSOFT $3050(\mathrm{GmbH}$ Greisinger electrónico) para Windows. En el análisis de los datos, las fases de meseta y peaks de presión, presiones diferenciadas y media bajo la curva fueron calculadas. Las fases de meseta incluyeron una oscilación de presión menor a $5 \mathrm{mbar} / \mathrm{s}$ y la duración de al menos $5 \mathrm{~s}$, a fin de distinguir las fases de meseta y la duración de la deglución normal (Kieser et al., 2008). Los peaks se definieron como un cambio en la presión de al menos $5 \mathrm{mbar} / \mathrm{s}$ en 2 segundos, y un segundo cambio (disminución) de al menos $5 \mathrm{mbar} / \mathrm{s}$ dentro de los $5 \mathrm{~s}$ después del inicio del cambio. Se describieron las frecuencias de peaks y etapas de meseta, así como duración y magnitud de las fases de meseta.

\section{RESULTADOS}

Un total de 15 pacientes fueron evaluados correctamente. En ambos métodos de medición, el 54\% de pacientes presentó patrones similares de presión intraoral en los compartimientos, el resto fue irregular. Los distintos cambios de presión producidos durante las fases de prueba con el escudo oral, se observan en la gráfica de la Figura $2 \mathrm{~A}$ y $\mathrm{B}$.

En la Fase B1 de condición abierta, el EIO se mantuvo con una presión constante entre los 0 y 4 $\mathrm{mBar}$, por lo cual se produce una meseta positiva durante esta condición. EI ESP se mantiene con una presión constante entre los -1 a -3 mBar y produce una meseta negativa (Fig. 2B).

En la Fase B2 condición semiabierta, en el EIO se produce un peak cercano a los $10 \mathrm{mBar}$ y se mantiene una meseta entorno a esta presión al minuto si-
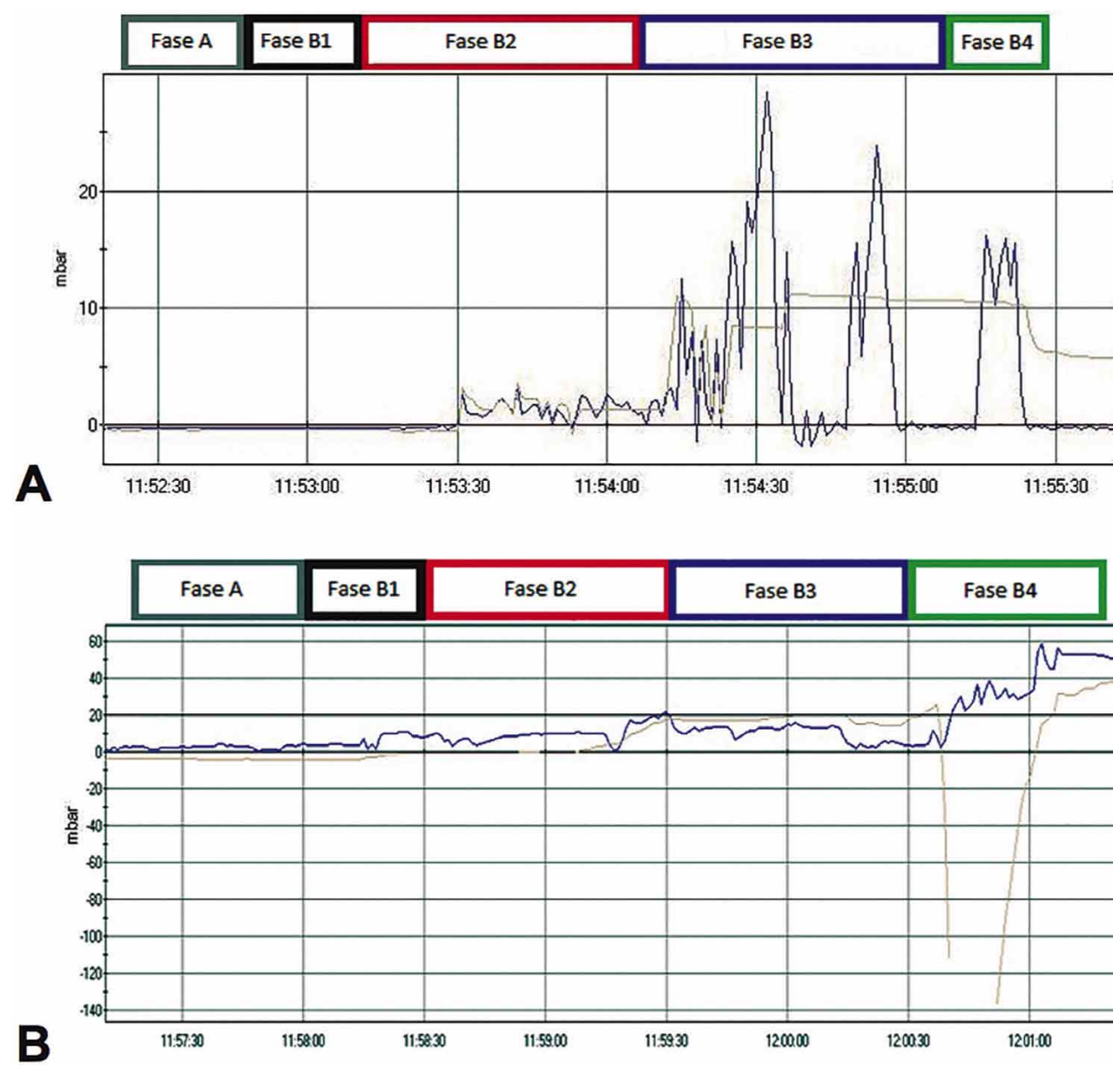

Fig. 2. A. Cambios de presión producidos durante las distintas fases biofuncionales de prueba con el método de escudo oral se observan en la gráfica. Nótese peaks de oscilación entre los 0 y 20 mBar en fase B3 y B4 del EIO (espacio interoclusal, línea azul), mientras que el ESP (espacio subpalatino, línea gris) muestra una aumento paulatino y constante hacia los $10 \mathrm{mBar}$ hasta el término de esta fase. B. Los cambios de presión intraoral con el escudo oral son similares entre la fase A y B3 a las observadas con el método de control. En la Fase B4 de medición de presión bajo deglución, el EIO presenta un peak desde 0 a 40 mBar mientras que ESP presenta un solo peak que varía entre 20 y $-140 \mathrm{mBar}$. 
guiente. El ESP no presenta variaciones significativas y se mantiene con una presión constante de 0 a -1 mBar (Fig. 2B).

En la Fase B3 condición cerrada, en el ElO se producen entre 4-5 peaks de oscilación que van entre 0 y 20 mBar. En el espacio ESP, se observa un aumento paulatino y constante durante los primeros 30 segundos, alcanzando hasta una presión de $20 \mathrm{mBar}$, posteriormente se mantiene en relación a los $20 \mathrm{mBar}$ de forma constante hasta el término de esta fase (Fig. 2B).

En la Fase B4 medición de presión bajo deglución, el EIO presenta un peak desde 0 a $40 \mathrm{mBar}$ y luego se presentan 3-4 peaks entre $20-40 \mathrm{mBar}$. El ESP presenta un solo peak desde 20 hasta $-40 \mathrm{mBar}$, una diferencia de más de 160 mBar (Fig. 2B).

\section{DISCUSIÓN}

A pesar de las alteraciones morfológicas propias de los adultos mayores edéntulos portadores de prótesis acrílicas, que modifican la función y competencia neuromuscular, se demostró la existencia de presiones intraorales negativas mediante el uso del dispositivo tipo escudo oral, principalmente en la fase de deglusión, estabilizando el equilibrio biofuncional del ESP, aún sin la adecuada estructura alveolar y dentaria, por lo que su reestablecimiento terapéutico es posible.

Observamos que el $46 \%$ de los sujetos evaluados presentó patrones irregulares principalmente en la fase de deglusión y la condición cerrada, lo que puede ser atribuido a falta de coordinación labial y lingual en las distintas fases o la poca adaptación al escudo intraoral, ya que su uso no fue contínuo, ni el paciente reeducado para su uso en el tiempo. Esto evidencia que disfunciones orofaciales, como la falta de equilibrio y establecimiento de presiones intraorales negativas, dependen de la actividad neuromuscular, donde externamente sobre el arco dentario las estructuras musculares de labios y mejillas están ejerciendo de manera sostenida una presión simétrica, e internamente, la lengua con igual persistencia ejerce una función recíproca y genera el estado de equilibrio (Ruan et al., 2005) y compartimientos biofuncionales intraorales (Moss \& Salentijn; Knösel et al.). El modelo que subyace esta teoría del equilibrio, se basa en el supuesto de que las fuerzas activas neuromusculares son responsables del equilibrio de la posición denta- ria y viceversa (Wein et al., 1988), lo que se logra mediante un balance entre la presión atmosférica positiva (extraoral) y una una presión negativa (intraoral), semejante a la presión intrapulmonar que posibilita un respiración balanceada.

Nuestros registros mostraron que en condición abierta (fase B1), no existe una variación considerable respecto a la presión atmosférica, indicando que la formación de los compartimentos de cierre no ocurren espontáneamente, similar a los descrito por Engelke et al. (2011)

En la condición semiabierta (fase B2), el ESP presentó una meseta negativa lo cual indicaría una formación temporal de un compartimiento cerrado, mientras que el IOS presenta una presión positiva, lo cual no difiere de manera significativa respecto de la condición abierta; esta condición puede ser explicada porque el escudo oral dificultaría el cierre del compartimiento vestibular, resultado similar a lo observado por Knösel et al., donde la pérdida dentaria y ósea no pueden superadas por el escudo.

En la condición cerrada (fase B3), la lengua se encuentra en posición postural y se produce la deglución involuntaria y respiración (Engelke et al., 2011). En el ESP se produjo un aumento de la presión lo cual indicaría un aumento de la actividad del paladar blando y lengua; respecto al EIO se producen una serie de variaciones de presiones en rangos levemente inferiores que las producidas en el ESP, lo que puede ser provocado por la influencia del escudo oral o la incordinación labial en la deglución que no genera un cierre (Engelke et al., 2007). Sin embargo la información obtenida indica que los dos compartimientos actúan parcialmente como unidades independientes.

En la deglución (fase B4), el ESP presenta un peak negativo de $160 \mathrm{mBar}$, lo cual indicaría la actividad lingual forzada; en el EIO también se produce un cierre y peak de $40 \mathrm{mBar}$, lo cual indicaría una menor actividad muscular de la zona perioral respecto a la lengua, demostrando la presencia de dos unidades de espacios funcionales separados (Engelke et al., 2007). La fuerte presión negativa obtenida, es similar a la descrita experimentalmemte por Fränkel (1967), así como a generadas al utilizar un escudo oral (Engelke et al., 2006, 2007), lo que en grupos desdentados puede ser similar a las generadas por los niños cuando realizan la succión digital y lactancia, demostrando la funcionalidad, influencia y participación de los músculos periorales y la lengua. 
Engelke et al. (2006) proponen que en una posición oral cerrada de descanso, junto con una adecuada posición de la lengua generando presión negativa intraoral, puede representar un mecanismo fundamental de la estabilización física del velo del paladar y la lengua y a su vez, puede reducir la cantidad de actividad neuromuscular necesaria para el mantenimiento de la permeabilidad de las vías respiratorias. Además, al examinar los movimientos del velo del paladar y la lengua durante la deglución, existe una posición de contacto inmediato de la lengua con el paladar duro, la que permite formar un sello velo-lingual después de la deglución, contribuyendo a la estabilización de la lengua en la cavidad oral (Engelke \& Hoch, 1994).

Los hallazgos reportados permiten postular el uso de terapias para entrenar a desdentados y posibilitar la recuperación de los espacios biofuncionales y mejorar su funcionalidad neuromuscular. Esto puede ser logrado mediante maniobras de reposicionamiento lingual descrita por Engelke (2003), con la formación voluntaria de presión negativa intraoral (cerrada posición de reposo) mediante una respiración exclusivamente nasal. Espacios biofuncionales generados en un estado cerrado a la presión subatmosférica, sirven como factor de estabilización biomecánico que generan distintas presiones intraorales, no solo a expensas de la actividad neuromuscular de las estructuras orofaciales, sino que también a las posturas orales biofuncionales, las que finalmente determinan el equilibrio biomecánico de las fuerzas por elementos externos e internos, observados normalmente en presencia de dientes (Engelke et al., 2007, 2010), pero que también se pueden encontrar y generar de manera estable en pacientes edéntulos.

\section{CONCLUSIÓN}

La formación de dos compartimientos cerrados con variaciones en la presión intraoral pueden ser confirmados en pacientes edéntulos, donde sólo el espacio subpalatino principalmente muestra cambios de presión negativa. Además, durante de la deglución pasiva se producen algunas mesetas de presión negativa las cuales podrían ser sostenidas en el tiempo. Estos patrones de presión intraoral en espacios biofuncionaless se observan en más del $50 \%$ de los casos, lo que permitiría realizar tratamientos que permitan su total reestablecimiento, mediante terapias de reposicionamiento lingual.

\section{AGRADECIMIENTOS}

Los autores agradecen a la Dirección de Investigación de la Universidad de La Frontera por su financiamiento a través del proyecto DIUFRO DI12-0051.

FUENTES, R.; ENGELKE, W.; FUENTES, C.; SOTO, N. \& CANTÍN, M. Determination of intraoral pressures on biofunctional compartments in edentulous patients. Int. J. Odontostomat., 8(2):151-157, 2014.

ABSTRACT: The aim was to describe the different intraoral pressures that occur in the vestibular interocclusal space (IOS) and subpalatal space (SPS) under different oral functions in adult patients with maxillary edentulism. A descriptive and cross-sectional study was conducted. We selected 15 patients at the Dental Clinic of the Dentistry Faculty (CODA), Universidad de la Frontera (Temuco, Chile). Intraoral pressure measurement was performed using a digital manometer GMH 3156, by methods of cannulas (control) and oral shield. A calibration measurement was made for 15 minutes; then four functional measurements were performed: B1 (open-mouth condition, 0-25 s), B2 (semi-open compartment condition, 35-85 s), B3 (closed compartments condition; 95-145 s) and B4 (swallowing, 150-165 s). With the oral shield, the B1 phase in the IOS showed a constant pressure between 0-4 mBar and SPS between -1 and $-3 \mathrm{mBar}$. In B2 phase, the IOS reached $10 \mathrm{mBar}$ and SPS between 0 and $-1 \mathrm{mBar}$. Phase B3 OIS oscillations showed between 0 and $20 \mathrm{mBar}$, and the SPS increased to 20 mbar. In B4 phase, the IOS showed a peak of 40 mbar, while the SPS showed a peak from 20 to -140 mbar. The formation of two closed compartments with negative intra-oral pressure also occurs in edentulous patients, where the SPS shows greater negative pressure changes than IOS. Regular patterns of intra-oral pressure in biofunctional compartments were observed in over $50 \%$ of cases.

KEY WORDS: edentulous patients, intra-oral pressure, negative pressure, biofunctional model, functional intra-oral compartments. 
FUENTES, R.; ENGELKE, W.; FUENTES, C.; SOTO, N. \& CANTÍN, M. Determinación de presiones intraorales en compartimientos biofuncionales de pacientes desdentados totales. Int. J. Odontostomat., 8(2):151-157, 2014.

\section{REFERENCIAS BIBLIOGRÁFICAS}

Abrams, I.N. Oral Muscle Pressures. Angle Orthod., 33(33):83-104, 1963.

Archer, S. Y. \& Vig, P. S. Effects of head position on intraoral pressures in Class I and Class II adults. Am. J. Orthod., 87(4):311-8, 1985.

Engelke, W. \& Hoch, G. Simultaneous electromagnetic articulography and video endoscopy. A case contribution to the objective diagnosis of the velopharyngeal sphincter. Fortschr. Kieferorthop., 55(6):297-303, 1994.

Engelke, W. Ein Manöver zur Positionierung der Zunge am Gaumen. Sprache Stimme Gehör, 27(4):171-5, 2003.

Engelke, W.G.; Mendoza, M. \& Repetto, G. Preliminary radiographic observations of the tongue-repositioning manoeuvre. Eur. J. Orthod., 28(6):618-23, 2006.

Engelke, W.; Repetto, G.; Mendoza-Gaertner, M. \& Knoesel, M. Functional treatment of snoring using oral shields in conjunction with the tongue repositioning manoevre. Int. J. Odontostomat., 1(2):133-9, 2007.

Engelke, W.; Engelhardt, W.; Mendoza-Gärtner, M.; Deccó, O.; Barrirero, J. \& Knösel, M. Functional treatment of snoring based on the tongue-repositioning manoeuvre. Eur. J. Orthod., 32(5):490-5, 2010.

Engelke, W.; Jung, K. \& Knösel, M. Intra-oral compartment pressures: a biofunctional model and experimental measurements under different conditions of posture. Clin. Oral Investig., 15(2):165-76, 2011.

Fränkel, R. Die Dynamik des interokklusalen Unterdruckes. Dtsch. Zahnärztl. Z., 22:282, 1967.

Jung, K.; Engelke, W. \& Knösel, M. Statistical signal processing methods for intraoral pressure curve analysis in orthodontics. Eur. J. Orthod., 34(4):437-41, 2012.

Knösel, M.; Jung, K.; Kinzinger, G.; Bauss, O. \& Engelke, W. A controlled evaluation of oral screen effects on intraoral pressure curve characteristics. Eur. J. Orthod., 32(5):535-41, 2010.

Kieser, J.; Singh, B.; Swain, M.; Ichim, I.; Waddell, J.N.; Kennedy, D.; Foster, K. \& Livingstone, V. Measuring intraoral pressure: adaptation of a dental appliance allows measurement during function. Dysphagia, 23(3):237-43, 2008.

Moss, M.L. \& Salentijn, L. The capsular matrix. Am. J. Orthod., 56(5):474-90, 1969.
Proffit, W. R. Equilibrium theory revisited: factors influencing position of the teeth. Angle Orthod., 48(3):175-85, 1978.

Ruan, W. H.; Chen, M. D.; Gu, Z. Y.; Lu, Y.; Su, J. M. \& Guo, $\mathrm{Q}$. Muscular forces exerted on the normal deciduous dentition. Angle Orthod., 75(5):785-90, 2005.

Wein, B.; Alzen, G.; Tolkdorff, T.; Bockler, R.; Klajman, S. \& Huber, W. Computerized sonographic imaging of tongue motility using pseudo 3-dimensional reconstruction. Ultraschall. Med., 9(2):95-7, 1988.

Dirección para Correspondencia:

Dr. Ramón Fuentes Fernández

Facultad de Odontología

Universidad de la Frontera

Manuel Montt 112

Casilla 54-D

Temuco, CHILE

Email: ramon.fuentes@ufrontera.cl

Recibido: 13-03-2014

Aceptado: 28-05-2014 